\title{
The synthesis of tetrafluorinated aminosugars
}

Clément Q. Fontenelle, Graham J. Tizzard, and Bruno Linclau*

Chemistry, University of Southampton, Highfield, Southampton SO17 1BJ

Email: Bruno Linclau - bruno.linclau@soton.ac.uk

* Corresponding author

Graphical abstract

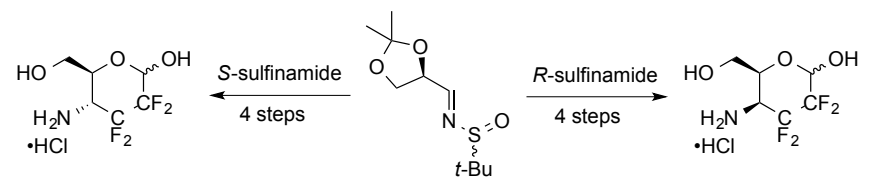




\title{
The synthesis of tetrafluorinated aminosugars
}

\author{
Clément Q. Fontenelle, Graham J. Tizzard, and Bruno Linclau* \\ Chemistry, University of Southampton, Highfield, Southampton SO17 1BJ \\ Email: Bruno Linclau - bruno.linclau@soton.ac.uk \\ * Corresponding author
}

\begin{abstract}
The synthesis of two tetrafluorinated 4-aminosugars, 4-amino-2,3,4-trideoxy-2,2,3,3-tetrafluoroD-erythro-hexopyranose hydrochloride $(7 \cdot \mathbf{H C I})$ and 4-amino-2,3,4-trideoxy-2,2,3,3-tetrafluoro-Dthreo-hexopyranose hydrochloride $(8 \cdot \mathbf{H C l})$, is described. The amino group in $\alpha$-position of a $\mathrm{CF}_{2}\left(\mathrm{CF}_{2}\right)$ group is proposed as a mimic for the hydrogen bond accepting capacity of an alcohol group in an unfluorinated sugar. The synthesis of the two sugars was achieved in 4 steps each from the sulfinylimine diastereoisomers of D-glyceraldehyde.
\end{abstract}

Keywords fluorine; carbohydrate; fluorosugar, fluorination, aminosugar

\section{Introduction}

Fluorination of carbohydrates is a popular strategy to investigate carbohydrate binding epitopes $^{[1]}$ and enzyme mechanism, ${ }^{[2]}$ or to stabilize glycosidic bonds, ${ }^{[2,3]}$ and indeed a vast number of fluorinated carbohydrates and their glycosides have been synthesized for these purposes. ${ }^{[4]}$ While the replacement of $\mathrm{CHOH}$ with $\mathrm{CHF}$ (or $\mathrm{CF}_{2}$ ) has as main consequence that the hydrogen bond donating capacity at that position is lost, the electronic properties of the remaining hydroxyl groups can also undergo substantial changes. With respect to protein binding, the change in hydrogen bond donating and accepting properties of these alcohol groups could have significant additional effects. While these properties are influenced by the fluorine electronegativity, there are other factors that play a role, such as intramolecular hydrogen bonding of the $\mathrm{OH}$ group with the fluorine atom and hyperconjugation effects, both of which depend on relative stereochemistry. ${ }^{[5]}$

With regard to alcohol hydrogen bond acceptor capacity, it is instructive to compare a relevant parameter, $\mathrm{p} K_{\mathrm{BHX}}$, which refers to the equilibrium of the acceptor with a standard hydrogen bond 
donor ( $p$-fluorophenol). ${ }^{[6]}$ Clearly, the hydrogen bond acceptor capacity of the alcohol group in trifluoroethanol $\mathbf{2}$ is reduced compared to that of ethanol $\mathbf{1}$ to such an extent that it cannot be considered a hydrogen bond acceptor any more (Table 1). A similar decrease is seen by comparing ethylamine 3 and 2,2,2-trifluoroethylamine 4 . Nevertheless, the $\mathrm{p} K_{\mathrm{BH}}$ value for 4 is relatively close to that of $\mathbf{1}$, so it can be proposed that a $\beta$-trifluorinated (or difluorinated) amine is a reasonable mimic for a regular alcohol, if hydrogen bond acceptor properties are concerned.

Table 1. Influence of trifluoromethylation on alcohol and amine hydrogen bond acceptor capacity.

\begin{tabular}{lclc} 
& $\mathrm{p} K_{\mathrm{BHX}}$ & & $\mathrm{p} K_{\mathrm{BHX}}$ \\
\hline $\mathrm{CH}_{3} \mathrm{CH}_{2} \mathrm{OH}(1)$ & 1.02 & $\mathrm{CH}_{3} \mathrm{CH}_{2} \mathrm{NH}_{2}(3)$ & 2.17 \\
$\mathrm{CF}_{3} \mathrm{CH}_{2} \mathrm{OH}(2)$ & -0.28 & $\mathrm{CF}_{3} \mathrm{CH}_{2} \mathrm{NH}_{2}(4)$ & 0.71 \\
\hline
\end{tabular}

The design of carbohydrate-based analogues with greater affinity to carbohydrate-processing proteins is of interest for use as probes or therapeutics. ${ }^{[7]}$ We have an interest in investigating polyfluorination of carbohydrates as a strategy for increasing the typically low protein-

carbohydrate binding affinities. Polyfluorination introduces a hydrophobic moiety, thus causing beneficial hydrophobic desolvation upon binding, ${ }^{[8]}$ yet the individual polar $\mathrm{C}-\mathrm{F}$ bonds retain the capacity for attractive interactions with electropositive protein residues. ${ }^{[9]}$ The combination of these effects has been coined "polar hydrophobicity". ${ }^{[10]}$ In order to retain chiral alcohol groups in the carbohydrate ring, which were deemed important for binding selectivity, we have focused on the synthesis of sugars containing a medium-size hydrophobic moiety such as 2,3-dideoxy2,2,3,3-tetrafluorinated carbohydrates, including "tetrafluorinated glucose" (2,3-dideoxy-2,2,3,3tetrafluoro-D-erythro-hexopyranose) 5 (Figure 1) and -galactose (2,3-dideoxy-2,2,3,3tetrafluoro-D-threo-hexopyranose) 6. ${ }^{[11]}$ It was shown that these structures retain the conventional carbohydrate shape, ${ }^{[12]}$ and 6 was found to be a weak substrate of the enzyme galactose oxidase. ${ }^{[13]}$ A successful inhibitor of the mycobacterial enzyme UDP-Gal mutase, based on a tetrafluorinated galactofuranose sugar, has been recently reported. ${ }^{[14]}$

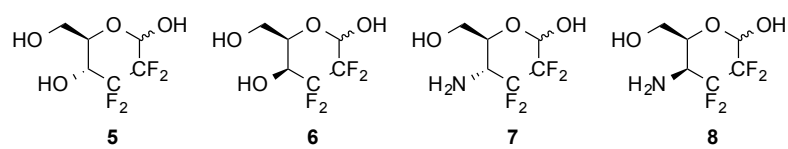

Figure 1. Tetrafluorinated sugars with the proposed aminosugar analogues. 
With the above discussion in mind, the hydrogen bond acceptor capacity of the 4-OH groups in 5 and $\mathbf{6}$ will be very low, and hence the corresponding tetrafluorinated aminosugars $\mathbf{7}$, a glucose analogue, and $\mathbf{8}$, a galactose analogue, became a focus for their synthesis and investigations. In addition, a further interest in their synthesis stems from the known interesting biological activities of aminosugars and their derivatives, ${ }^{[15]}$ with only a small number of fluorinated aminosugar derivatives reported. ${ }^{[16]}$

Herein we report the synthesis of $\mathbf{7}$ and $\mathbf{8}$.

\section{Results and discussion}

The synthesis of the 4-aminosugars was envisaged by reaction of the lithiated fluorinated building block $\mathbf{B}$ with a chiral glyceraldehyde derived sulfinylimine $\mathbf{A}$. The absolute configuration of the auxiliary was expected to control the configuration of the newly formed chiral centre. ${ }^{[17,18]}$

The corresponding reactants $9 / 10$ and 11 are known ${ }^{[18]}$ or commercially available. After the addition reaction, diol deprotection and alkene ozonolysis would give the fluorinated aminosugar.

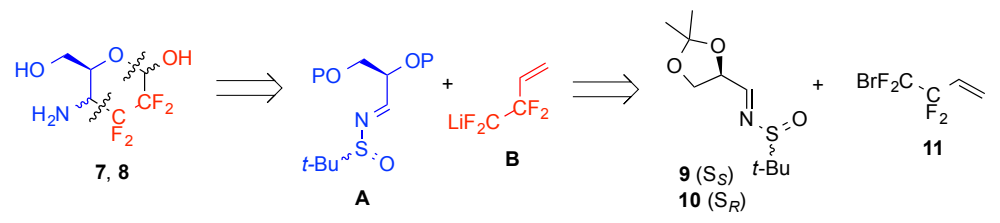

Scheme 1. Retrosynthetic analysis

The plan benefitted from important literature precedence, in that Konno had not only demonstrated that reagent $\mathbf{B}$ could be formed and cleanly reacted with electrophiles, but that it also reacted with the sulfinylimine derived from benzaldehyde (a 9:1 diastereomeric ratio was reported). ${ }^{[19]}$ The ozonolysis/pyranose ring formation had also been demonstrated in an efficient synthesis of 2,3-dideoxy-2,2,3,3-tetrafluorinated glucose $\mathbf{5}$ and galactose $\mathbf{6}$ by the same group. ${ }^{[20]}$

The synthesis of $\mathbf{7}$ is shown in Scheme 2. Following Konno's conditions, using the $(S)$ configured sulfinylimine auxiliary, a $78 \%$ yield was achieved for the coupling reaction as a $92: 8$ mixture of diastereoisomers. However, under these conditions, the limiting reagent is 
bromotetrafluorobutene 11 , with no less than 2.4 equiv of sulfinylimine used. Given 3 steps are used to obtain the sulfinylimines, we chose to reduce the relative amount of this substrate, in order to increase the isolated quantity of adducts $12 / 13$. Hence, reducing the number of equivalents of 9 to 1.2, a reduced $61 \%$ (isolated) yield was obtained for $12 / 13$, in a $4: 96$ ratio of diastereoisomers, but in a larger absolute quantity than would have been obtained under Konno's conditions. Interestingly, under these modified conditions the formation of a minor byproduct was observed which, despite isolation in pure form was not possible, could be assigned as $14(\sim 3 \%$, Scheme 3$)$. The presence of the terminal methyl group and the alkene C$\mathrm{H}$ were clearly observed in the ${ }^{1} \mathrm{H}$ NMR spectrum, and the ${ }^{3} \mathrm{~J}_{\mathrm{H}-\mathrm{F}}$ value of $36.5 \mathrm{~Hz}$ indicated a $\mathrm{Z}$ substituted fluoroalkene. The ${ }^{19} \mathrm{~F}$ NMR spectrum showed three resonances, including one geminal $\mathrm{CF}_{2}$ group as obvious from a large coupling constant. This type of byproduct, not reported by Konno, presumably arose from nucleophilic attack of MeLi to the alkene moiety in $12 / 13$ via $S_{N} 2$ ' fashion as shown. ${ }^{[21]}$

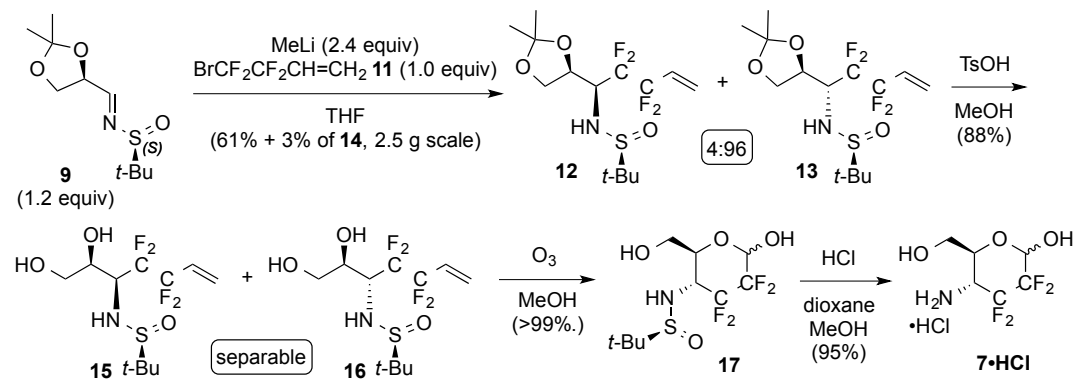

Scheme 2. Synthesis of the glucose analogue 7 .

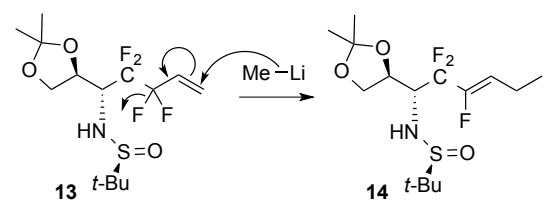

Scheme 3. Proposed identity and formation of the byproduct 14. 
High-yielding acetonide hydrolysis allowed separation of the diastereoisomers, leading to the desired product as a single diastereoisomer 16 in $88 \%$ isolated yield. Ozonolysis and amine auxiliary removal gave the 4-deoxy-4-amino glucose derivative 7 in high yield, as the hydrochloric acid salt. Interestingly, the precipitated salt was obtained as pure $\alpha$ anomer,

The similar synthesis of the corresponding galactose isomer is shown in Scheme 3. Now the $(R)$-configured sulfinylimine auxiliary is used for the reaction with lithiated 1,1,2,2tetrafluorobutene, leading to a separable mixture of adducts 18 and 19. The MeLi $S_{N} 2$ ' adduct was again observed as minor isomer (not shown). Diol deprotection and ozonolysis led to the pyranose 21 in excellent yield. The removal of the auxiliary proved cumbersome, in that for this compound, precipitation as the hydrochloride salt was not possible. This resulted in an incomplete separation from the sulfinate ester byproduct. Any attempt of purification by chromatography proved unsuccessful, leading to a complex mixture. Protection of the 4-amino and 6-hydroxy groups as $\mathrm{Cbz}$ allowed the purification but subsequent hydrogenolysis resulted in obtaining a similar complex mixture. As methyl tert-butanesulfinate is somewhat volatile (52 ${ }^{\circ} \mathrm{C} / 16$ torr), purification was attempted by co-evaporating with $\mathrm{MeOH}$ carefully keeping the temperature below $40{ }^{\circ} \mathrm{C}$ to avoid apparition of impurities. This proved only partially successful, but after dissolving the salt in water, the impurity could largely be removed by extraction with $\mathrm{Et}_{2} \mathrm{O}$. Hence, $\mathbf{8} \bullet \mathrm{HCl}$ was obtained in excellent yield.

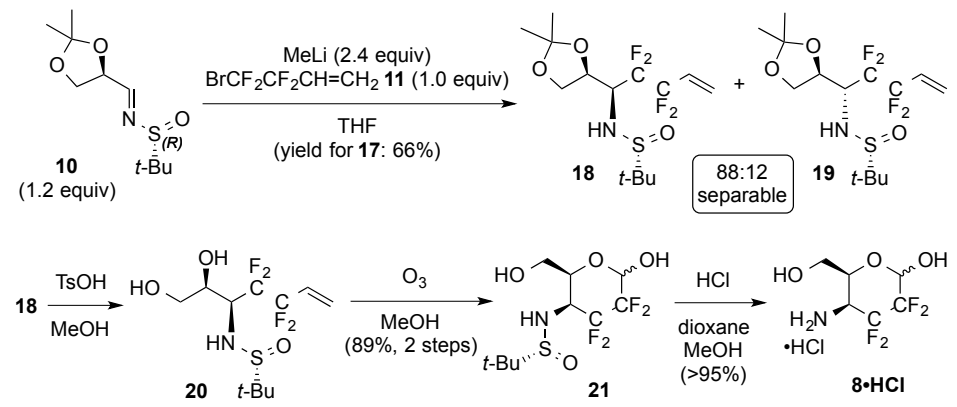

Scheme 3. Synthesis of the galactose analogue 8.

The relative stereochemistry of the obtained products could be deduced from X-ray crystallographic analysis of 17 (Figure 2). With the $S_{S}$-configuration of the auxiliary and the C5 configuration from the starting material retained in the product, the gluco configuration at C4 is
Linclau B.J. 10/7/2014 17:07

Deleted: , which unfortunately did not yield suitable crystals for X-ray diffraction analysis 
evident, as is the ${ }^{4} C_{1}$ conformation. This was also confirmed in solution by ${ }^{13} \mathrm{C}$ NMR analysis, in that the ${ }^{2} J_{\text {C4-F }}$ values were $19 \mathrm{~Hz}$ for both fluorine atoms (for both anomers), indicating that the electronegative substituent at the 4-position is equatorial. ${ }^{[22]}$ While we have not been able to crystallise 21, a similar NMR analysis showed ${ }^{2} J_{\mathrm{C} 4-\mathrm{F}}$ values of around 30 and $19 \mathrm{~Hz}$ (for both anomers), indicating an axial electronegative substituent at $\mathrm{C} 4$. The ${ }^{13} \mathrm{C}$ NMR of the fully deprotected aminosugars $\mathbf{7}$ and $\mathbf{8}$ showed similar values.

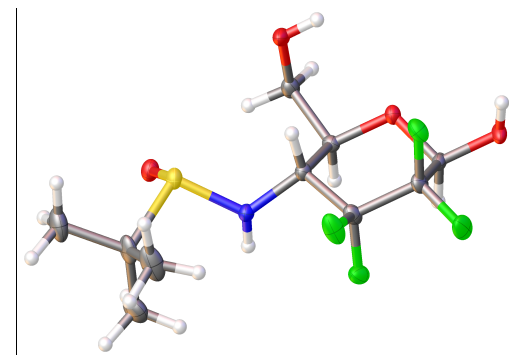

Figure 2. X-ray crystallographic analysis of $\beta-17$.

The ${ }^{2} J_{\text {C4-F }}$ values mentioned above for both the gluco and galacto configured structures also indicated that they existed in the pyranose form in solution. This was unambiguously shown by HMBC analysis of the aminosugars 7 and 8 (see supporting information). Irradiation of the anomeric proton led to a cross peak to $\mathrm{C} 5$ (and not to $\mathrm{C} 4$, which would represent the possible iminosugar isomer).

Interestingly, $\mathbf{7} \cdot \mathbf{H C l}$ solidified as the pure $\alpha$-anomer, though no crystals suitable for X-ray crystallography could be obtained. The anomeric equilibrium in $\mathrm{CD}_{3} \mathrm{OD}$ consisted of a 75:25 $\alpha / \beta$ mixture of anomers. The anomeric equilibrium for the galacto configured $8 \cdot \mathrm{HCl}$ in $\mathrm{CD}_{3} \mathrm{OD}$ was $54: 46 \alpha / \beta$.

The observed stereochemical outcome of the addition reactions to give 12/13 and 18/19 clearly demonstrated that the absolute configuration of the sulfinylimine auxiliary determined the stereochemical course of the reaction. The formation of the major isomers is consistent with an open transition state as shown in Figure 3 (left). ${ }^{[23]}$ The difference in stereoselectivity of the addition of $\mathbf{1 1}$ to $\mathbf{9}$ or $\mathbf{1 0}$ can be explained by the additional influence of the glyceraldehyde stereogenic centre. 

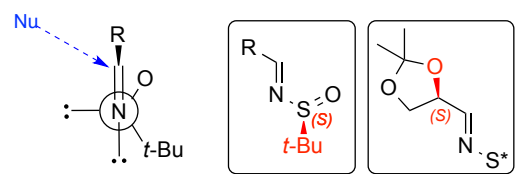

Si-face attack

等,

(Cornforth-Evans)

Figure 3. Explanation for the diastereoselectivity of the addition reactions.

According to the Cornforth-Evans model of stereoselection ${ }^{24]}$ (or the polar Felkin Anh model, ${ }^{[25]}$ not shown), the S-glyceraldehyde configuration induces Si-face attack (Figure 3, right). This is also the imine face that the $S$-configured sulfinylimine auxiliary makes available for reaction, according to the open transition state shown. Hence, in the $\left(S, S_{S}\right)$ combination (9), both stereoelements lead to a matched stereoinduction, resulting in a 96:4 ratio of products. In contrast, the stereoinduction in the $\left(S, R_{\mathrm{S}}\right)$ combination (10) shows a mismatch, leading to a reduced $88: 12$ ratio of products. ${ }^{[26]}$

\section{Conclusion}

The $\beta, \beta$-difluorinated amino moiety is proposed as a mimic for alcohol groups with regard to hydrogen bond accepting capacity. This led to tetrafluorinated aminosugars $\mathbf{7}$ and 8 as analogues of interest in the context of our investigations involving polyfluorinated carbohydrates. A short synthesis of these aminosugars is described with the addition of a lithiated tetrafluorobutene building block to a glyceraldehyde sulfinylimine as key step.

\section{Experimental}

4.1 (2S, 3S, $S_{S}$ )-1,2-Isopropylidenedioxy-3-(tert-butylsulfinylamino)-4,4,5, 5-tetrafluorohept-6-ene

(12) and $\left(2 S, 3 R, S_{S}\right)$-1,2-isopropylidenedioxy-3-(tert-butylsulfinylamino)-4,4,5,5-tetrafluorohept6-ene (13)

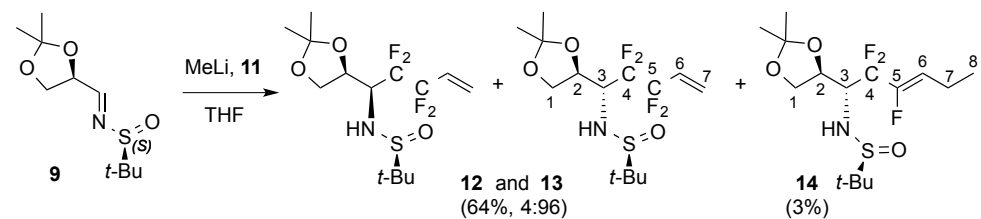


To a solution of sulfinylimine $9\left(2.5 \mathrm{~g}, 10.7 \mathrm{mmol}, 1.2\right.$ equiv) in THF (40 mL) at $-78{ }^{\circ} \mathrm{C}$ was added bromotetrafluorobutene 11 (1.14 mL, $8.93 \mathrm{mmol}, 1.0$ equiv). After $10 \mathrm{~min}$, MeLi (1.6 M in $\mathrm{Et}_{2} \mathrm{O}, 13.4 \mathrm{~mL}, 21.4 \mathrm{mmol}, 2.4$ equiv) was added dropwise over $30 \mathrm{~min}$ and the reaction mixture was stirred for another $1.5 \mathrm{~h}$. The reaction was quenched with saturated $\mathrm{NH}_{4} \mathrm{Cl}$ aq. $(25 \mathrm{~mL})$, diluted with $\mathrm{H}_{2} \mathrm{O}(15 \mathrm{~mL})$ and extracted with $\mathrm{Et}_{2} \mathrm{O}(3 \times 75 \mathrm{~mL})$. The combined organic layers were dried $\left(\mathrm{MgSO}_{4}\right)$, filtered and concentrated in vacuo to give a crude mixture of diastereoisomers ( $d r$ 97:3). Purification via column chromatography (petroleum ether/EtOAc $60: 40$ to $50: 50)$ afforded $1.96 \mathrm{~g}(5.43 \mathrm{mmol}, 61 \%)$ of a mixture of diastereoisomers $12 / 13$ along with $0.098 \mathrm{~g}(0.27 \mathrm{mmol}, 3 \%)$ of 14 as an off-white solid. $R_{f} 0.23$ (petroleum ether 40-60 ${ }^{\circ} \mathrm{C} /$ EtOAc 60:40). IR (neat) 3219 (w, br), 2985 (m), $1371(\mathrm{~m}), 1112$ (s), 1056 (s) cm ${ }^{-1} .{ }^{1} \mathrm{H}$ NMR $\left(400 \mathrm{MHz}, \mathrm{CDCl}_{3}\right) \delta 6.14-5.80\left(\mathrm{~m}, 4 \mathrm{H}, \mathrm{H}-7_{\text {trans }}+\mathrm{H}-6\right.$, major and minor), $5.71\left(\mathrm{~d},{ }^{3} J_{\mathrm{HH}}=10.6 \mathrm{~Hz}\right.$, $1 \mathrm{H}, \mathrm{H}-7_{\text {cis }}$, major), 5.70 (d, ${ }^{3} J_{\mathrm{HH}}=10.9 \mathrm{~Hz}, 1 \mathrm{H}, \mathrm{H}-7_{\text {cis }}$, minor), 4.61-4.54 (m, 2H, H-2, major and minor), 4.20-4.08 (m, 1H, H-3, major), 4.08-3.99 (m, 3H, $\mathrm{H}-1_{\mathrm{a}+\mathrm{b}}$, major and $\mathrm{H}-1_{\mathrm{a}}$, minor), 3.95 (d, ${ }^{3} J_{\mathrm{HH}}=10.2 \mathrm{~Hz}, 1 \mathrm{H}, \mathrm{NH}$, minor), 3.78 (dd, ${ }^{2} J_{\mathrm{HH}}=8.2,{ }^{3} J_{\mathrm{HH}}=6.1 \mathrm{~Hz}, 1 \mathrm{H}, \mathrm{H}-1_{\mathrm{b}}$, minor), 3.82-3.70 (m, $1 \mathrm{H}, \mathrm{H}-3$, minor), 3.68 (d, ${ }^{3} \mathrm{~J}_{\mathrm{HH}}=5.4 \mathrm{~Hz}, 1 \mathrm{H}, \mathrm{NH}$, major), 1.55 (s, 3H, $\mathrm{CH}_{3, \mathrm{Pr}}$, major), 1.45 (s, $3 \mathrm{H}, \mathrm{CH}_{3, \mathrm{PPr}}$, minor), 1.32 (s, $6 \mathrm{H}, \mathrm{CH}_{3, \mathrm{PPr}}$, major and minor), 1.24 (s, 9H, $\mathrm{CH}_{3, \mathrm{tBu}}$, minor), 1.22 (s, $9 \mathrm{H}, \mathrm{CH}_{3, \mathrm{tBu}}$, major) ppm. ${ }^{13} \mathrm{C} \mathrm{NMR}\left(101 \mathrm{MHz}, \mathrm{CDCl}_{3}\right) \delta 126.4$ (t, ${ }^{2} J_{\mathrm{CF}}=24.2 \mathrm{~Hz}, \mathrm{C}-6$, minor), 126.0 (t, ${ }^{2} J_{\mathrm{CF}}=24.2 \mathrm{~Hz}, \mathrm{C}-6$, major), 124.5 (t, ${ }^{3} J_{\mathrm{CF}}=9.5 \mathrm{~Hz}, \mathrm{C}-7$, major), 124.3 (t, ${ }^{3} J_{\mathrm{CF}}=9.5 \mathrm{~Hz}, \mathrm{C}-$ 7, minor), 115.9 (tt, ${ }^{1} J_{\mathrm{CF}}=256.1,{ }^{2} J_{\mathrm{CF}}=36.6 \mathrm{~Hz}, \mathrm{CF}_{2}$, major), 115.5 (tt, ${ }^{1} J_{\mathrm{CF}}=248.8,{ }^{2} J_{\mathrm{CF}}=35.1 \mathrm{~Hz}$, $\mathrm{CF}_{2}$, major), 110.1 ( $\mathrm{C}_{\mathrm{q}, \mathrm{Pr}}$, minor), 109.7 (C $\mathrm{q}_{\mathrm{i} \text { Pr }}$, major), 72.7 (C-2, minor), 72.6 (C-2, major), 66.5 (C-1, minor), 64.6 (d, ${ }^{4} J_{\mathrm{CF}}=4.4 \mathrm{~Hz}, \mathrm{C}-1$, major), 58.4 (t, ${ }^{2} \mathrm{~J}_{\mathrm{CF}}=23.4 \mathrm{~Hz}, \mathrm{C}-3$, minor), 57.8 (t, ${ }^{2} J_{\mathrm{CF}}=21.3 \mathrm{~Hz}, \mathrm{C}-3$, major), 57.6 ( $\mathrm{C}_{\mathrm{q}, \mathrm{tBu}}$, minor), 56.7 ( $\mathrm{C}_{\mathrm{q}, \mathrm{tBu}}$, major$), 26.2\left(\mathrm{CH}_{3, \mathrm{Pr} r}\right.$, minor $), 25.8$ ( $\mathrm{CH}_{3, \mathrm{PPr}}$, major), 24.34 ( $\mathrm{CH}_{3, \mathrm{PPr}}$, major), $24.25\left(\mathrm{CH}_{3, \mathrm{PPr}}\right.$, minor), $22.5\left(\mathrm{CH}_{3, \mathrm{BBu}}\right.$, minor $), 22.3\left(\mathrm{CH}_{3, \mathrm{BBu}}\right.$, major) ppm (2 $\times \mathrm{CF}_{2}$, minor not visible). ${ }^{19} \mathrm{~F} \mathrm{NMR}\left(282 \mathrm{MHz}, \mathrm{CDCl}_{3}\right) \delta-109.8$ (dd, ${ }^{2} J_{\mathrm{FF}}=279.2$, $\mathrm{J}=8.6 \mathrm{~Hz}, 1 \mathrm{~F}$, minor), $-111.8--112.8(\mathrm{~m}, 1 \mathrm{~F}$, major), $-112.4--113.5$ ( $\mathrm{m}, 1 \mathrm{~F}$, major), $-113.1-$ -114.2 (m, 1F, major), -118. 7 (ddd, ${ }^{2} J_{\mathrm{FF}}=279.4, J=17.2,{ }^{3} J_{\mathrm{FF}}=4.4 \mathrm{~Hz}, 1 \mathrm{~F}$, minor), -120.1 (app. ddt, ${ }^{2} J_{\mathrm{FF}}=281.5, J=16.1,7.5 \mathrm{~Hz}, 1 \mathrm{~F}$, major) ppm (2 $\times \mathrm{F}$, minor overlap with major). MS (ESI+) $(\mathrm{m} / \mathrm{z}) 425(\mathrm{M}+\mathrm{Na}+\mathrm{MeCN})^{+}$. HRMS $(\mathrm{MS}+)$ for $\mathrm{C}_{14} \mathrm{H}_{23} \mathrm{~F}_{4} \mathrm{NNaO}_{3} \mathrm{~S}(\mathrm{M}+\mathrm{Na})^{+}$calcd 384.1227 , found 384.1233.

Selected data for the MeLi $S_{N}$ ' byproduct (2S,3R, $\left.S_{S}, Z\right)-1,2-$ Isopropylidenedioxy-3-(tertbutylsulfinylamino)-4,4,5-trifluorooct-5-ene (14): ${ }^{1} \mathrm{H}$ NMR (400 MHz, $\left.\mathrm{CDCl}_{3}\right) \delta 5.47$ (dt, $\left.{ }^{3} J_{\mathrm{HF}, \text { trans }}=36.5,{ }^{3} \mathrm{~J}_{\mathrm{HH}}=7.6 \mathrm{~Hz}, 1 \mathrm{H}, \mathrm{H}-6\right), 2.28-2.15(\mathrm{~m}, 2 \mathrm{H}, \mathrm{H}-7), 1.03\left(\mathrm{t},{ }^{3} \mathrm{~J}_{\mathrm{HH}}=7.5 \mathrm{~Hz}, 3 \mathrm{H}, \mathrm{H}-8\right)$ ppm. ${ }^{13} \mathrm{C}$ NMR $\left(101 \mathrm{MHz}, \mathrm{CDCl}_{3}\right) \delta 16.8\left(\mathrm{~d},{ }^{4} \mathrm{~J}_{\mathrm{CF}}=4.4 \mathrm{~Hz}, \mathrm{C}-7\right), 13.1$ (s, C-8) ppm. ${ }^{19} \mathrm{~F}$ NMR $(376$ 
$\left.\mathrm{MHz}, \mathrm{CDCl}_{3}\right) \delta-108.1$ (dddt, ${ }^{2} J_{\mathrm{FF}}=265.0,{ }^{3} J_{\mathrm{FF}}=14.8, \mathrm{~J}=10.8,2.2 \mathrm{~Hz}, 1 \mathrm{~F}, \mathrm{~F}-4$ ), -110.8 (app. dt, ${ }^{2} J_{\mathrm{FF}}=265.0, J=13.3 \mathrm{~Hz}, 1 \mathrm{~F}, \mathrm{~F}-4$ '), $-132.0--132.2(\mathrm{~m}, 1 \mathrm{~F}, \mathrm{~F}-5) \mathrm{ppm} . \mathrm{MS}(\mathrm{ESI}+)(\mathrm{m} / \mathrm{z}) 358$ $(\mathrm{M}+\mathrm{H})^{+}$. HRMS (MS+) for $\mathrm{C}_{15} \mathrm{H}_{27} \mathrm{~F}_{3} \mathrm{NO}_{3} \mathrm{~S}(\mathrm{M}+\mathrm{H})^{+}$calcd 358.1658, found 358.1663. The C3 stereochemistry is assumed.

$4.2\left(2 S, 3 S, S_{S}\right)$-3-(tert-Butylsulfinylamino)-4,4,5,5-tetrafluorohept-6-ene-1,2-diol

(15) and

$\left(2 S, 3 R, S_{S}\right)$ - 3-(tert-butylsulfinylamino)-4,4,5,5-tetrafluorohept-6-ene-1,2-diol (16)

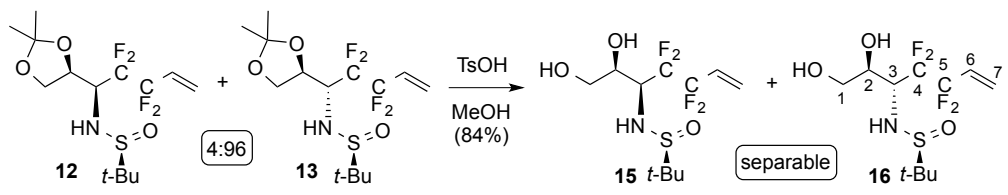

The 4:96 mixture of $12 / 13$ (2.06 g, $5.70 \mathrm{mmol}$, 1 equiv) was dissolved in $\mathrm{MeOH}$ (60 mL). PTSA (196 mg, $1.14 \mathrm{mmol}, 0.2$ equiv) was added, and the solution stirred for $23 \mathrm{~h}$, and then quenched with sat. aq. $\mathrm{NaHCO}_{3}(30 \mathrm{~mL}) . \mathrm{H}_{2} \mathrm{O}(30 \mathrm{~mL})$ was added and the mixture was extracted with EtOAc $(3 \times 120 \mathrm{~mL})$. The combined organic layers were washed with $\mathrm{H}_{2} \mathrm{O}(10 \mathrm{~mL})$, dried $\left(\mathrm{Na}_{2} \mathrm{SO}_{4}\right)$, filtered and concentrated. Purification via column chromatography (petroleum ether/acetone $70: 30$ to $50: 50)$ afforded $1.607 \mathrm{~g}(5.00 \mathrm{mmol}, 88 \%)$ of the pure major diasteroisomer 16 as a yellow syrup. $R_{f} 0.51$ (petroleum ether $40-60{ }^{\circ} \mathrm{C} /$ acetone $60: 40$ ). $[\alpha]_{D}$ +68.3 (c $0.204, \mathrm{CHCl}_{3}, 25^{\circ} \mathrm{C}$ ). IR (neat) 3362 (m, br), 3243 (m, br), 2962 (w), 1102 (s), 1068 (s), 1039 (s) $\mathrm{cm}^{-1} .{ }^{1} \mathrm{H}$ NMR $\left(400 \mathrm{MHz}, \mathrm{CDCl}_{3}\right) \delta 6.12-5.83\left(\mathrm{~m}, 2 \mathrm{H}, \mathrm{H}-6+\mathrm{H}-7_{\text {trans }}\right), 5.73$ (d, ${ }^{3} J_{\mathrm{HH}}=10.6$ $\left.\mathrm{Hz}, 1 \mathrm{H}, \mathrm{H}-7_{\text {cis }}\right), 5.57$ (d, $\left.{ }^{3} \mathrm{~J}_{\mathrm{HH}}=9.5 \mathrm{~Hz}, 1 \mathrm{H}, \mathrm{NH}\right), 4.83$ (d, $\left.{ }^{3} \mathrm{~J}_{\mathrm{HH}}=10.3 \mathrm{~Hz}, 1 \mathrm{H}, \mathrm{OH}-2\right), 4.21-4.07(\mathrm{~m}$, $1 \mathrm{H}, \mathrm{H}-3), 4.07-3.91\left(\mathrm{~m}, 3 \mathrm{H}, \mathrm{H}-1_{\mathrm{a}+\mathrm{b}}, \mathrm{H}-2\right), 3.48-3.35(\mathrm{~m}, 1 \mathrm{H}, \mathrm{OH}-1), 1.27\left(\mathrm{~s}, 9 \mathrm{H}, \mathrm{CH}_{3, \mathrm{BBu}}\right) \mathrm{ppm}$. ${ }^{13} \mathrm{C}$ NMR $\left(101 \mathrm{MHz}, \mathrm{CDCl}_{3}\right) \delta 126.3\left(\mathrm{t},{ }^{2} J_{\mathrm{CF}}=24.4 \mathrm{~Hz}, \mathrm{C}-6\right), 124.3\left(\mathrm{t},{ }^{3} \mathrm{~J}_{\mathrm{CF}}=9.5 \mathrm{~Hz}, \mathrm{C}-7\right), 66.1$ (C2), $65.0(\mathrm{C}-1), 62.8\left(\mathrm{t},{ }^{2} \mathrm{~J}_{\mathrm{CF}}=22.6 \mathrm{~Hz}, \mathrm{C}-3\right), 56.7\left(\mathrm{C}_{\mathrm{q}, \mathrm{BBu}}\right), 22.6\left(\mathrm{CH}_{3, \mathrm{BBu}}\right) \mathrm{ppm}\left(2 \times \mathrm{CF}_{2}\right.$ not visible $)$. ${ }^{19} \mathrm{~F}$ NMR $\left(376 \mathrm{MHz}, \mathrm{CDCl}_{3}\right) \delta-112.7\left(\mathrm{dd},{ }^{2} J_{\mathrm{FF}}=264.0, J_{\mathrm{HF}}=11.3 \mathrm{~Hz}, 1 \mathrm{~F}\right),-113.9\left(\mathrm{dd},{ }^{2} J_{\mathrm{FF}}=264.0\right.$, $\left.J_{\mathrm{HF}}=11.3 \mathrm{~Hz}, 1 \mathrm{~F}\right),-118.9\left(\mathrm{dd},{ }^{2} J_{\mathrm{FF}}=277.4, J_{\mathrm{HF}}=13.0 \mathrm{~Hz}, 1 \mathrm{~F}\right),-119.7\left(\mathrm{dd},{ }^{2} J_{\mathrm{FF}}=277.4, J_{\mathrm{HF}}=15.6\right.$ $\mathrm{Hz}, 1 \mathrm{~F}) \mathrm{ppm}$. MS (ESI+) (m/z) $385(\mathrm{M}+\mathrm{Na}+\mathrm{MeCN})^{+}$. HRMS (MS+) for $\mathrm{C}_{11} \mathrm{H}_{19} \mathrm{~F}_{4} \mathrm{NNaO}_{3} \mathrm{~S}(\mathrm{M}+$ $\mathrm{Na})^{+}$calcd 344.0914 , found 344.0915 .

A sample was purified by HPLC to obtain the minor isomer 15 in pure form (hexane/acetone 70:30). $\mathrm{R}_{\mathrm{f}} 0.31$ (petroleum ether $40-60{ }^{\circ} \mathrm{C} /$ acetone 60:40). $[\alpha]_{\mathrm{D}}-0.866$ (c $0.289, \mathrm{CHCl}_{3}, 25{ }^{\circ} \mathrm{C}$ ). IR (neat) $3368(\mathrm{~m}), 3280(\mathrm{~m}), 1107$ (s), 1053 (s), 1036 (s) $\mathrm{cm}^{-1} .{ }^{1} \mathrm{H}$ NMR $\left(400 \mathrm{MHz}, \mathrm{CDCl}_{3}\right) \delta$ 6.13-5.98 (m, 1H, H-6), 5.94-5.87 (m, 1H, H-7 trans), $5.74\left(\mathrm{~d},{ }^{3} J_{\mathrm{HH}}=10.9 \mathrm{~Hz}, 1 \mathrm{H}, \mathrm{H}-7_{\text {cis }}\right), 4.36(\mathrm{~d}$, $\left.{ }^{3} J_{H H}=9.0 \mathrm{~Hz}, 1 \mathrm{H}, \mathrm{NH}\right), 4.28(\mathrm{qd}, J=5.8,2.9 \mathrm{~Hz}, 1 \mathrm{H}, \mathrm{H}-2), 4.08-3.96(\mathrm{~m}, 1 \mathrm{H}, \mathrm{H}-3), 3.71$ (dd, 
$\left.{ }^{2} J_{\mathrm{HH}}=11.6,{ }^{3} \mathrm{JHH}_{\mathrm{HH}}=6.0 \mathrm{~Hz}, 1 \mathrm{H}, \mathrm{H}-1_{\mathrm{a}}\right), 3.65\left(\mathrm{dd},{ }^{2} \mathrm{~J}_{\mathrm{HH}}=11.6,{ }^{3} \mathrm{JHH}_{\mathrm{HH}}=6.4 \mathrm{~Hz}, 1 \mathrm{H}, \mathrm{H}-1_{\mathrm{b}}\right), 3.13\left(\mathrm{~d},{ }^{3} \mathrm{~J}_{\mathrm{HH}}=5.3\right.$ $\mathrm{Hz}, 1 \mathrm{H}, \mathrm{OH}-2), 3.02\left(\mathrm{t},{ }^{3} \mathrm{JHH}_{\mathrm{HH}}=6.6 \mathrm{~Hz}, 1 \mathrm{H}, \mathrm{OH}-1\right), 1.26 \mathrm{ppm}\left(\mathrm{s}, 9 \mathrm{H}, \mathrm{CH}_{3, \mathrm{tBu}}\right) \mathrm{ppm} .{ }^{13} \mathrm{C} \mathrm{NMR}(101$ $\left.\mathrm{MHz}, \mathrm{CDCl}_{3}\right) \delta 126.3\left(\mathrm{t},{ }^{2} \mathrm{~J}_{\mathrm{CF}}=24.5 \mathrm{~Hz}, \mathrm{C}-6\right), 124.4\left(\mathrm{t},{ }^{3} \mathrm{~J}_{\mathrm{CF}}=9.5 \mathrm{~Hz}, \mathrm{C}-7\right), 115.8$ (tt, ${ }^{1} \mathrm{~J}_{\mathrm{CF}}=249.6$, $\left.{ }^{2} J_{\mathrm{CF}}=35.9 \mathrm{~Hz}, \mathrm{CF}_{2}\right), 116.7\left(\mathrm{tt},{ }^{1} J_{\mathrm{CF}}=256.1,{ }^{2} J_{\mathrm{CF}}=35.5 \mathrm{~Hz}, \mathrm{CF}_{2}\right), 68.6(\mathrm{C}-2), 63.1$ (C-1), 57.7 $\left(\mathrm{C}_{\mathrm{q}, \mathrm{Bu}}\right), 54.7\left(\mathrm{t},{ }^{2} \mathrm{~J}_{\mathrm{CF}}=22.7 \mathrm{~Hz}, \mathrm{C}-3\right), 22.4\left(\mathrm{CH}_{3, \mathrm{Bu}}\right)$ ppm. ${ }^{19} \mathrm{~F} \mathrm{NMR}\left(282 \mathrm{MHz}, \mathrm{CDCl}_{3}\right) \delta-110.4$ (dd, $\left.{ }^{1} J_{\mathrm{FF}}=279.4, J=10.7 \mathrm{~Hz}, 1 \mathrm{~F}, \mathrm{CFF}\right),-112.7\left(\mathrm{~d}, J=11.8 \mathrm{~Hz}, 2 \mathrm{~F}, \mathrm{CF}_{2}\right.$ ), -117.0 (dd, ${ }^{1} J_{\mathrm{FF}}=279.4$, $J=16.1 \mathrm{~Hz}, 1 \mathrm{~F}, \mathrm{CF} \underline{)}) \mathrm{ppm}$.

$4.3\left(S_{S}\right)$-4-(tert-Butylsulfinylamino)-2,3,4-trideoxy-2,2,3,3-tetrafluoro-D-erythro-hexopyranose (17)

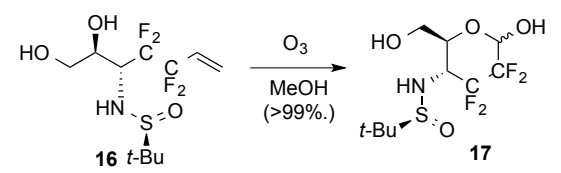

Ozone was bubbled through a solution of $16(1.60 \mathrm{~g}, 4.98 \mathrm{mmol})$ in $\mathrm{MeOH}(50 \mathrm{~mL})$ until TLC showed complete consumption of the starting material (15 min). $\mathrm{O}_{2}$ was bubbled through to remove excess ozone (10 $\mathrm{min}$ ) and then, $\mathrm{Me}_{2} \mathrm{~S}(1.83 \mathrm{~mL}, 24.9 \mathrm{mmol}, 5$ equiv) was added and the reaction mixture was allowed to warm to $\mathrm{rt}$ and concentrated to afford $1.56 \mathrm{~g}(4.83 \mathrm{mmol}$, $97 \%$ ) of the pure aminosugar derivative 17 , which solidified as the pure $\beta$-anomer. At equilibrium in $C_{3} \mathrm{OD}$, a $60: 40 \alpha / \beta$ mixture of anomers is obtained. $\mathrm{R}_{\mathrm{f}} 0.23$ (petroleum ether 40 $60^{\circ} \mathrm{C} /$ acetone $\left.60: 40\right)$. $[\alpha]_{\mathrm{D}}+97.6$ (c $0.469, \mathrm{CH}_{3} \mathrm{OH}, 26^{\circ} \mathrm{C}$, at anomeric equilibrium). IR (neat) 3245 (m), 2985 (w), 1303 (m), 1151 (m), 1037 (s) cm ${ }^{-1} .{ }^{1} \mathrm{H}$ NMR (400 MHz, CD ${ }_{3} \mathrm{OD}$ ) $\delta 5.23$ (dd, $\left.{ }^{3} J_{\mathrm{HF}}=7.8,5.5 \mathrm{~Hz}, 1 \mathrm{H}, \mathrm{H}-1 \alpha\right), 4.90\left(\mathrm{dd},{ }^{3} J_{\mathrm{HF}}=15.5, J_{\mathrm{HF}}=2.9 \mathrm{~Hz}, 1 \mathrm{H}, \mathrm{H}-1 \beta\right), 4.29-4.18(\mathrm{~m}, 1 \mathrm{H}, \mathrm{H}-$ $5 \alpha)$, 3.98-3.76 (m, 6H, H-4a, H-4ß, $2 \times \mathrm{H}-6 \alpha, 2 \times \mathrm{H}-6 \beta), 3.76-3.69(\mathrm{~m}, 1 \mathrm{H}, \mathrm{H}-5 \beta), 1.26(\mathrm{~s}, 18 \mathrm{H}$, $\left.\mathrm{CH}_{3, \mathrm{tBu}}, \alpha+\mathrm{CH}_{3, \mathrm{tBu}}, \beta\right)$ ppm. ${ }^{13} \mathrm{C} \mathrm{NMR}\left(101 \mathrm{MHz}, \mathrm{CD}_{3} \mathrm{OD}\right) \delta 92.9$ (ddd, ${ }^{2} J_{\mathrm{CF}}=26.4,{ }^{2} J_{\mathrm{CF}}=19.4$, $\left.{ }^{3} J_{\mathrm{CF}}=2.6 \mathrm{~Hz}, \mathrm{C}-1 \beta\right), 92.8\left(\mathrm{dd},{ }^{2} J_{\mathrm{CF}}=36.6,{ }^{2} J_{\mathrm{CF}}=26.3 \mathrm{~Hz}, \mathrm{C}-1 \alpha\right), 75.2\left(\mathrm{~d}, J_{\mathrm{CF}}=2.9 \mathrm{~Hz}, \mathrm{C}-5 \beta\right), 70.6$ $\left(\mathrm{d}, J_{\mathrm{CF}}=4.4 \mathrm{~Hz}, \mathrm{C}-5 \alpha\right), 61.4(\mathrm{C}-6 \beta), 61.3(\mathrm{C}-6 \alpha), 59.2\left(\mathrm{t},{ }^{2} J_{\mathrm{CF}}=18.7 \mathrm{~Hz}, \mathrm{C}-4 \beta\right), 59.0\left(\mathrm{t},{ }^{2} J_{\mathrm{CF}}=17.6\right.$ $\mathrm{Hz}, \mathrm{C}-4 \alpha), 58.6\left(2 \times \mathrm{C}_{\mathrm{q}, \mathrm{tBu}}\right), 23.2\left(\mathrm{CH}_{3, \mathrm{tBu}}, \alpha\right), 23.2\left(\mathrm{CH}_{3, \mathrm{tBu}}, \beta\right) \mathrm{ppm}\left(2 \times \mathrm{CF}_{2}, \alpha+\beta\right.$ not visible $) .{ }^{19} \mathrm{~F}$ NMR (376 MHz, CD $\left.{ }_{3} \mathrm{OD}\right) \delta-121.3--122.3(\mathrm{~m}, 1 \mathrm{~F}, \mathrm{F \alpha}),-125.2$ (dddd, ${ }^{2} J_{\mathrm{FF}}=258.4, J=21.7$, 15.6, $6.9 \mathrm{~Hz}, 1 \mathrm{~F}, \mathrm{F \alpha}),-125.9--126.8(\mathrm{~m}, 1 \mathrm{~F}, \mathrm{F \alpha}),-128.2\left(\mathrm{dt},{ }^{2} J_{\mathrm{FF}}=259.2, J=16.5 \mathrm{~Hz}, 1 \mathrm{~F}, \mathrm{~F} \beta\right)$, -129.1 (dq, ${ }^{2} J_{\mathrm{FF}}=259.2, \mathrm{~J}=10.4 \mathrm{~Hz}, 1 \mathrm{~F}, \mathrm{~F} \beta$ ), -135.8 (ddd, ${ }^{2} J_{\mathrm{FF}}=265.3, J=15.2,11.7 \mathrm{~Hz}, 1 \mathrm{~F}, \mathrm{F \alpha}$ ), $-138.5\left(\mathrm{dt},{ }^{2} J_{\mathrm{FF}}=257.5,12.6 \mathrm{~Hz}, 1 \mathrm{~F}, \mathrm{~F} \beta\right),-140.8--141.7(\mathrm{~m}, 1 \mathrm{~F}, \mathrm{~F} \beta) \mathrm{ppm}$. MS (ESI+) (m/z) 387 $(\mathrm{M}+\mathrm{Na}+\mathrm{MeCN})^{+}$. HRMS (MS+) for $\mathrm{C}_{10} \mathrm{H}_{17} \mathrm{~F}_{4} \mathrm{NNaO}_{4} \mathrm{~S}(\mathrm{M}+\mathrm{Na})^{+}$calcd 346.0707, found 346.0706 . 


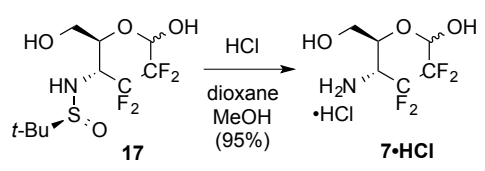

A solution of 17 (700 mg, $2.17 \mathrm{mmol}, 1$ equiv) in $\mathrm{MeOH}(1.65 \mathrm{~mL})$ and $4 \mathrm{M} \mathrm{HCl}$ in dioxane (1.1 $\mathrm{mL}, 4.33 \mathrm{mmol}, 2$ equiv) was stirred at $\mathrm{rt}$ for $1 \mathrm{~h}$ then evaporated in vacuo to near dryness. $\mathrm{Et}_{2} \mathrm{O}$ $(10 \mathrm{~mL})$ was added in order to precipitate the hydrochloride salt and the supernatant was removed. The solid was washed once more with $\mathrm{Et}_{2} \mathrm{O}(10 \mathrm{~mL})$ then dried under vacuum to yield $525 \mathrm{mg}(2.05 \mathrm{mmol}, 95 \%)$ of the $7 \cdot \mathbf{H C l}$ as a white solid consisting only of $\alpha$-anomer. At equilibrium in $\mathrm{CD}_{3} \mathrm{OD}$, a $75: 25 \alpha / \beta$ mixture of anomers is obtained. $[\alpha]_{\mathrm{D}}+52.7$ (c $0.430, \mathrm{CH}_{3} \mathrm{OH}$, $26{ }^{\circ} \mathrm{C}$, at anomeric equilibrium). IR (neat) 3343 (m, br), 2888 (m, br), 1153 (s), 1111 (s), 1059 (s) $\mathrm{cm}^{-1}$. Data for the $\alpha$ anomer: ${ }^{1} \mathrm{H}$ NMR $\left(400 \mathrm{MHz}, \mathrm{CD}_{3} \mathrm{OD}\right) \delta 5.33\left(\mathrm{dd}, J_{\mathrm{HF}}=7.3,4.3 \mathrm{~Hz}, 1 \mathrm{H}, \mathrm{H}-\right.$ 1), 4.38 (dt, J=10.3, $3.5 \mathrm{~Hz}, 1 \mathrm{H}, \mathrm{H}-5), 4.07-3.93(\mathrm{~m}, 1 \mathrm{H}, \mathrm{H}-4), 3.85$ (dd, ${ }^{2} J_{\mathrm{HH}}=12.5,{ }^{3} J_{\mathrm{HH}}=4.3 \mathrm{~Hz}$, $1 \mathrm{H}, \mathrm{H}-6_{\mathrm{a}}$ ), $3.80\left(\mathrm{dd},{ }^{2} \mathrm{~J}_{\mathrm{HH}}=12.5,{ }^{3} \mathrm{~J}_{\mathrm{HH}}=3.7 \mathrm{~Hz}, 1 \mathrm{H}, \mathrm{H}-6_{\mathrm{b}}\right) \mathrm{ppm} .{ }^{13} \mathrm{C} \mathrm{NMR}\left(101 \mathrm{MHz}, \mathrm{CD}_{3} \mathrm{OD}\right) \delta$ 117.9-108.8 (2 × $\left.\mathrm{CF}_{2}\right), 92.6\left(\mathrm{dd},{ }^{2} J_{\mathrm{CF}}=35.6,26.0 \mathrm{~Hz}, \mathrm{C}-1\right), 67.9\left(\mathrm{~d}, J_{\mathrm{CF}}=2.2 \mathrm{~Hz}, \mathrm{C}-5\right), 61.7$ (C-6), $52.5\left(\mathrm{t},{ }^{2} J_{\mathrm{CF}}=19.1 \mathrm{~Hz}, \mathrm{C}-4\right) \mathrm{ppm} .{ }^{19} \mathrm{~F}$ NMR (376 MHz, CD $\left.{ }_{3} \mathrm{OD}\right) \delta-121.4--122.5(\mathrm{~m}, 1 \mathrm{~F}),-124.0$ $--125.8(\mathrm{~m}, 2 \mathrm{~F}),-137.1\left(\mathrm{dt},{ }^{2} J_{\mathrm{FF}}=267.6, J=12.4 \mathrm{~Hz}, 1 \mathrm{~F}\right) \mathrm{ppm}$. Unambiguous resonances for the $\beta$ anomer: $1 \mathrm{H}$ NMR $\left(400 \mathrm{MHz}, \mathrm{CD}_{3} \mathrm{OD}\right) \delta 5.06\left(\mathrm{~d}, \mathrm{~J}_{\mathrm{HF}}=14.3 \mathrm{~Hz}, 1 \mathrm{H}, \mathrm{H}-1\right) .{ }^{13} \mathrm{C} \mathrm{NMR}(101 \mathrm{MHz}$, $\left.\mathrm{CD}_{3} \mathrm{OD}\right) \delta 72.4$ (s, C-5), 61.8 (s, C-6). $\left.{ }^{19} \mathrm{~F} \mathrm{NMR} \mathrm{(376} \mathrm{MHz,} \mathrm{CD} \mathrm{CD}_{3}\right) \delta-127.8$ (t, J=12.1 Hz, 2F), $-139.6\left(\mathrm{dt},{ }^{2} J_{\mathrm{FF}}=260.1, \mathrm{~J}=11.3 \mathrm{~Hz}, 1 \mathrm{~F}\right),-140.8$ (dd, $\left.{ }^{2} J_{\mathrm{FF}}=260.1, J=13.9 \mathrm{~Hz}, 1 \mathrm{~F}\right) \mathrm{ppm}$. MS (ESI+) $(m / z) 261(\mathrm{M}+\mathrm{H}+\mathrm{MeCN})^{+}$. HRMS (MS+) for $\mathrm{C}_{6} \mathrm{H}_{10} \mathrm{~F}_{4} \mathrm{NO}_{3}(\mathrm{M}+\mathrm{H})^{+}$calcd 220.0591, found 220.0590 .

$4.5\left(2 S, 3 S, R_{S}\right)$-1,2-Isopropylidenedioxy-3-(tert-butylsulfinylamino)-4,4,5,5-tetrafluorohept-6-ene (18)

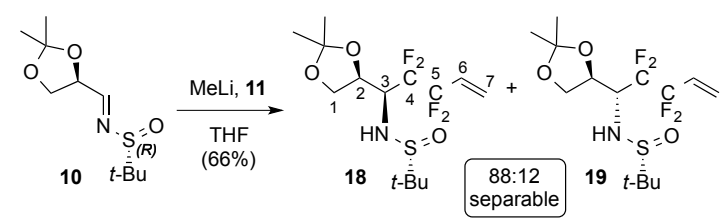

To a solution of sulfinylimine $10\left(0.52 \mathrm{~g}, 2.23 \mathrm{mmol}, 1.2\right.$ equiv) in THF at $-78{ }^{\circ} \mathrm{C}$ was added bromotetrafluorobutene $\left(0.236 \mathrm{~mL}, 1.86 \mathrm{mmol}, 1.0\right.$ equiv). After $10 \mathrm{~min}$, MeLi (1.6 $\mathrm{M}$ in $\mathrm{Et}_{2} \mathrm{O}, 2.8$ $\mathrm{mL}, 4.46 \mathrm{mmol}, 2.4$ equiv) was added over $45 \mathrm{~min}$ and the reaction mixture was stirred for 
another $1.5 \mathrm{~h}$. The reaction was quenched with saturated $\mathrm{NH}_{4} \mathrm{Cl}$ aq. (10 mL) and extracted with $\mathrm{Et}_{2} \mathrm{O}(3 \times 30 \mathrm{~mL})$. The combined organic layers were dried $\left(\mathrm{MgSO}_{4}\right)$, filtered and concentrated in vacuo to give a crude mixture of diastereoisomers 18 and 19 ( $d r$ 88:12). Purification via column chromatography (petroleum ether/EtOAc 75:25) afforded $442 \mathrm{mg}(1.22 \mathrm{mmol}, 66 \%)$ of pure 18 as a white solid. $R_{f} 0.29$ (petroleum Ether 40-60 ${ }^{\circ} \mathrm{C} / E t O A c ~ 70: 30$ ). [ $\left.\alpha\right]_{D}-77.6$ (c 0.502, $\left.\mathrm{CHCl}_{3}, 19{ }^{\circ} \mathrm{C}\right)$. IR (neat) 3347 (w), 2982 (w), 1189 (m), 1109 (s), 1073 (s) cm ${ }^{-1} .{ }^{1} \mathrm{H}$ NMR (400 $\left.\mathrm{MHz}, \mathrm{CDCl}_{3}\right) \delta 5.98$ (ddd, $\left.{ }^{3} J_{\mathrm{HF}}=22.6,{ }^{3} J_{\mathrm{HH}, \text { trans }}=17.3,{ }^{3} J_{\mathrm{HH}, \text { cis }}=10.9 \mathrm{~Hz}, 1 \mathrm{H}, \mathrm{H}-6\right), 5.90-5.81(\mathrm{~m}, 1 \mathrm{H}$, $\left.\mathrm{H}-7_{\text {trans }}\right), 5.69$ (d, ${ }^{3} J_{\mathrm{HH}, \text { cis }}=10.9 \mathrm{~Hz}, 1 \mathrm{H}, \mathrm{H}-7_{\text {cis }}$ ), 4.54 (t, J=6.9 Hz, $\left.1 \mathrm{H}, \mathrm{H}-2\right), 4.31$ (d, J=7.3 Hz, $1 \mathrm{H}$, $\mathrm{NH}$ ), 4.17 (app. t, J=8.2 Hz, 1H, H-1 ${ }_{\mathrm{a}}$ ), 4.07 (dd, ${ }^{2} J_{\mathrm{HH}}=8.5,{ }^{3} J_{\mathrm{HH}}=6.7 \mathrm{~Hz}, 1 \mathrm{H}, \mathrm{H}-1_{\mathrm{b}}$ ), 3.82 (td, $J=12.9,7.6 \mathrm{~Hz}, 1 \mathrm{H}, \mathrm{H}-3), 1.46\left(\mathrm{~s}, 3 \mathrm{H}, \mathrm{CH}_{3, \mathrm{Pr}}\right), 1.37$ (s, 3H, $\left.\mathrm{CH}_{3, \mathrm{Pr}}\right), 1.25 \mathrm{ppm}\left(\mathrm{s}, 9 \mathrm{H}, \mathrm{CH}_{3, \mathrm{BBu}}\right)$ ppm. ${ }^{13} \mathrm{C}$ NMR $\left(101 \mathrm{MHz}, \mathrm{CDCl}_{3}\right) \delta 126.5\left(\mathrm{t},{ }^{2} \mathrm{~J}_{\mathrm{CF}}=24.2 \mathrm{~Hz}, \mathrm{C}-6\right), 124.0\left(\mathrm{t},{ }^{3} \mathrm{~J}_{\mathrm{CF}}=9.5 \mathrm{~Hz}, \mathrm{C}-7\right)$, $115.7\left(\mathrm{tt},{ }^{1} J_{\mathrm{CF}}=256.1,{ }^{2} J_{\mathrm{CF}}=35.1 \mathrm{~Hz}, \mathrm{CF}_{2}\right), 115.5\left(\mathrm{tt},{ }^{1} J_{\mathrm{CF}}=250.3,{ }^{2} J_{\mathrm{CF}}=35.1 \mathrm{~Hz}, \mathrm{CF}_{2}\right), 110.2$ $\left(\mathrm{C}_{\mathrm{q}, \mathrm{Pr}}\right), 71.3(\mathrm{C}-5), 66.2(\mathrm{C}-1), 56.7\left(\mathrm{C}_{\mathrm{q}, \mathrm{BBu}}\right), 56.1$ (t, $\left.{ }^{2} J_{\mathrm{CF}}=23.4 \mathrm{~Hz}, \mathrm{C}-3\right), 26.2\left(\mathrm{CH}_{3, \mathrm{Pr}}\right), 25.3$ $\left(\mathrm{CH}_{3, \mathrm{Pr}}\right), 22.6\left(\mathrm{CH}_{3, \mathrm{tBu}}\right) \mathrm{ppm} .{ }^{19} \mathrm{~F} \mathrm{NMR}\left(376 \mathrm{MHz}, \mathrm{CDCl}_{3}\right) \delta-112.3\left(\mathrm{dd},{ }^{2} \mathrm{~J}_{\mathrm{FF}}=265.3, \mathrm{~J}=11.3 \mathrm{~Hz}\right.$, 1F), -113.1 (dd, $\left.{ }^{2} J_{F F}=265.3, J=11.3 \mathrm{~Hz}, 1 \mathrm{~F}\right),-117.2\left(\mathrm{dd},{ }^{2} J_{\mathrm{FF}}=277.4, J=13.9 \mathrm{~Hz}, 1 \mathrm{~F}\right),-118.3$ $\left(\mathrm{ddd},{ }^{2} J_{\mathrm{FF}}=277.4, \mathrm{~J}=12.1,3.5 \mathrm{~Hz}, 1 \mathrm{~F}\right) \mathrm{ppm}$. MS (ESI+) $(\mathrm{m} / \mathrm{z}) 425(\mathrm{M}+\mathrm{Na}+\mathrm{MeCN})^{+}$. HRMS (MS+) for $\mathrm{C}_{14} \mathrm{H}_{23} \mathrm{~F}_{4} \mathrm{NNaO}_{3} \mathrm{~S}(\mathrm{M}+\mathrm{Na})^{+}$calcd 384.1227 , found 384.1230 .

The minor isomer $\left(2 S, 3 R, R_{S}\right)$-1,2-Isopropylidenedioxy-3-(tert-butylsulfinylamino)-4,4,5,5tetrafluorohept-6-ene 19 could be isolated along with the MeLi $\mathrm{S}_{\mathrm{N}} 2$ ' byproduct and some unknown impurity (53 mg, 76:9:15 ratio). Selected characterization data: $R_{f} 0.17$ (petroleum ether 40-60 ${ }^{\circ} \mathrm{C} /$ EtOAc 70:30). ${ }^{1} \mathrm{H}$ NMR $\left(400 \mathrm{MHz}, \mathrm{CDCl}_{3}\right) \delta$ 6.14-5.98 (m, 1H, H-6), 5.93-5.85 $\left(\mathrm{m},{ }^{3} J_{\mathrm{HH}, \text { trans }}=17.5 \mathrm{~Hz}, 1 \mathrm{H}, \mathrm{H}-7_{\text {trans }}\right), 5.73$ ( $\left.\mathrm{d},{ }^{3} \mathrm{~J}_{\mathrm{HH}, \mathrm{cis}}=10.9 \mathrm{~Hz}, 1 \mathrm{H}, \mathrm{H}-7_{\text {cis }}\right), 4.50-4.43(\mathrm{~m}, 1 \mathrm{H}, \mathrm{H}-2)$, 4.24-4.12 (m, 1H, H-3), 3.98 (app. t, J=7.5 Hz, 1H, H-1 a), 3.79 (app. t, J=7.8 Hz, 1H, H-1 b), 3.73 (d, $\left.{ }^{3} \mathrm{~J}_{\mathrm{HH}}=7.6 \mathrm{~Hz}, 1 \mathrm{H}, \mathrm{NH}\right), 1.40\left(\mathrm{~s}, 3 \mathrm{H}, \mathrm{CH}_{3, \mathrm{Pr}}\right.$ ), $1.32\left(\mathrm{~s}, 3 \mathrm{H}, \mathrm{CH}_{3, \mathrm{Pr}}\right), 1.24\left(\mathrm{~s}, 9 \mathrm{H}, \mathrm{CH}_{3, \mathrm{BBu}}\right) \mathrm{ppm} .{ }^{13} \mathrm{C}$ NMR (101 MHz, CDCl $\left.)_{3}\right) \delta 125.9$ (t, $\left.{ }^{2} J_{\mathrm{CF}}=24.2 \mathrm{~Hz}, \mathrm{C}-6\right), 124.9$ (t, $\left.{ }^{3} J_{\mathrm{CF}}=9.5 \mathrm{~Hz}, \mathrm{C}-7\right), 115.7$ (tt, $\left.{ }^{1} J_{\mathrm{CF}}=256.4,{ }^{2} J_{\mathrm{CF}}=35.6 \mathrm{~Hz}, \mathrm{CF}_{2}\right), 115.6\left(\mathrm{tt},{ }^{1} J_{\mathrm{CF}}=249.4,{ }^{2} J_{\mathrm{CF}}=33.7 \mathrm{~Hz}, \mathrm{CF}_{2}\right), 109.0\left(\mathrm{C}_{\mathrm{q}, \mathrm{Pr}}\right), 73.3(\mathrm{C}-$ 2), $64.8(\mathrm{C}-1), 57.5\left(\mathrm{t},{ }^{2} J_{\mathrm{CF}}=23.1 \mathrm{~Hz}, \mathrm{C}-3\right), 57.0\left(\mathrm{C}_{\mathrm{q}, \mathrm{BBu}}\right), 26.0\left(\mathrm{CH}_{3, \mathrm{Pr}}\right), 24.6\left(\mathrm{CH}_{3, \mathrm{Pr}}\right), 22.5$ $\left(\mathrm{CH}_{3, \mathrm{tBu}}\right)$ ppm. ${ }^{19} \mathrm{~F} \mathrm{NMR}\left(376 \mathrm{MHz}, \mathrm{CDCl}_{3}\right) \delta-111.3\left(\mathrm{~m},{ }^{2} J_{\mathrm{FF}}=264.9 \mathrm{~Hz}, 1 \mathrm{~F}\right),-113.3$ (ddt, $\left.{ }^{2} J_{\mathrm{FF}}=264.9, J=12.1,6.5 \mathrm{~Hz}, 1 \mathrm{~F}\right),-114.6$ (ddt, $\left.{ }^{2} J_{\mathrm{FF}}=278.5, J=12.6,5.6 \mathrm{~Hz}, 1 \mathrm{~F}\right),-117.4$ (ddt, $\left.{ }^{2} J_{\mathrm{FF}}=278.5, J=14.5,6.1 \mathrm{~Hz}, 1 \mathrm{~F}\right) \mathrm{ppm}$.

Selected data for the MeLi $S_{N}$ ' byproduct (2S,3S, $\left.R_{S}, Z\right)$-1,2-Isopropylidenedioxy-3-(tert$\chi_{\mathrm{O}}$ butylsulfinylamino)-4,4,5-trifluorooct-5-ene: ${ }^{1} \mathrm{H} \mathrm{NMR}\left(400 \mathrm{MHz}^{\mathrm{CDCl}} \mathrm{CDC}_{3} \delta\right.$

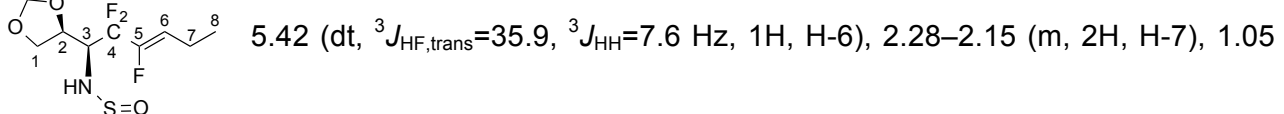
$S=O$ 
(t, $\left.{ }^{3} J_{\mathrm{HH}}=7.5 \mathrm{~Hz}, 3 \mathrm{H}, \mathrm{H}-8\right)$ ppm. ${ }^{13} \mathrm{C}$ NMR $\left(101 \mathrm{MHz}, \mathrm{CDCl}_{3}\right) \delta 16.7$ (d, $\left.{ }^{4} \mathrm{~J}_{\mathrm{CF}}=4.0 \mathrm{~Hz}, \underline{\mathrm{C}-7}\right), 13.0$ (C8) ppm. ${ }^{19} \mathrm{~F}$ NMR (376 MHz, $\left.\mathrm{CDCl}_{3}\right) \delta-107.2$ (app. dt, $\left.{ }^{2} J_{\mathrm{FF}}=264.4, \mathrm{~J}=13.9 \mathrm{~Hz}, 1 \mathrm{~F}, \mathrm{~F}-4\right),-112.8$ (app. dt, ${ }^{2} J_{F F}=264.4, J=13.9 \mathrm{~Hz}, 1 \mathrm{~F}, \mathrm{~F}-4$ ) $),-130.8$ (app. dt, ${ }^{3} J_{\mathrm{HF} \text {, trans }}=35.5, J=14.7 \mathrm{~Hz}, 1 \mathrm{~F}, \mathrm{~F}-3$ ) ppm. The stereochemistry at C3 is presumed.

4.6 (RS $\left(R_{S}\right.$-4-(tert-Butylsulfinylamino)-2,3,4-trideoxy-2,2,3,3-tetrafluoro-D-threo-hexopyranose (21)

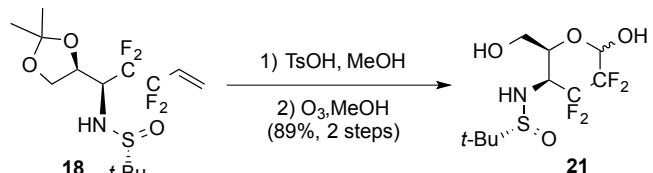

A mixture of sulfinamine 18 (435 mg, $1.20 \mathrm{mmol}, 1$ equiv) and PTSA (41 mg, $0.24 \mathrm{mmol}, 0.2$ equiv) in $\mathrm{MeOH}(10 \mathrm{~mL})$ was stirred for $13.5 \mathrm{~h}$ then quenched with sat. aq. $\mathrm{NaHCO}_{3}(3 \mathrm{~mL}) . \mathrm{H}_{2} \mathrm{O}$ $(12 \mathrm{~mL})$ was added and the mixture was extracted with EtOAc $(3 \times 30 \mathrm{~mL})$. The combined organic layers were washed with $\mathrm{H}_{2} \mathrm{O}(10 \mathrm{~mL})$, dried $\left(\mathrm{Na}_{2} \mathrm{SO}_{4}\right)$, filtered and concentrated to afford $383 \mathrm{mg}$ of the crude product 20. The latter was dissolved in $\mathrm{MeOH}(15 \mathrm{~mL})$ and ozone was bubbled through the solution until blue colour appeared (15 $\mathrm{min}$ ). $\mathrm{O}_{2}$ was bubbled through to remove excess ozone (10 $\mathrm{min})$ and then, $\mathrm{Me}_{2} \mathrm{~S}(0.44 \mathrm{~mL}, 6.0 \mathrm{mmol}, 5$ equiv) was added and the reaction mixture was allowed to warm to $\mathrm{rt}$ and concentrated. Purification via column

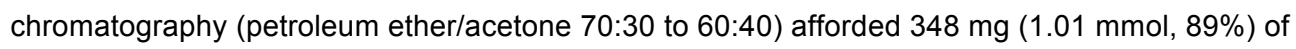
the pure aminosugar derivative $\mathbf{2 1}$, as a white solid enriched in $\beta$-anomer. At equilibrium in both acetone- $d_{6}$ and $C D_{3} \mathrm{OD}$, a 50:50 $\alpha / \beta$ mixture of anomers was obtained. $\mathrm{R}_{\mathrm{f}} 0.19$ (petroleum ether 40-60 ${ }^{\circ} \mathrm{C} /$ acetone 60:40). $[\alpha]_{\mathrm{D}}+20.0\left(\mathrm{c} 0.627, \mathrm{CH}_{3} \mathrm{OH}, 26{ }^{\circ} \mathrm{C}\right.$, at anomeric equilibrium). IR (neat) 3487 (w), 3287 (m), 2975 (w), 1041 (s), 1005 (s) cm ${ }^{-1} .{ }^{1} \mathrm{H}$ NMR (400 MHz, acetone-d $\left.)_{6}\right) \delta .36$

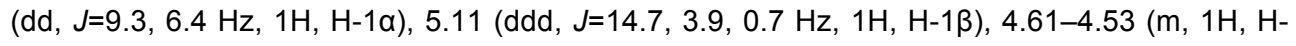
$5 \alpha), 4.51-4.41(\mathrm{~m}, 2 \mathrm{H}, \mathrm{NH \alpha}, \mathrm{NH} \beta), 4.35-4.16(\mathrm{~m}, 2 \mathrm{H}, 2 \times \mathrm{OH}-6), 4.16-4.03(\mathrm{~m}, 3 \mathrm{H}, \mathrm{H}-4 \alpha, \mathrm{H}-4 \beta$, $\mathrm{H}-5 \beta)$, 3.82-3.64 (m, 4H, $2 \times \mathrm{H}-6 \alpha, 2 \times \mathrm{H}-6 \beta), 1.26$ (s, 9H, tBu, $\beta), 1.25$ (s, 9H, tBu, $\alpha)$ ppm. ${ }^{13} \mathrm{C}$ NMR (101 MHz, acetone- $\left.d_{6}\right) \delta 93.2$ (ddd, ${ }^{2} J_{\mathrm{CF}}=27.1,19.8,{ }^{3} J_{\mathrm{CF}}=3.7 \mathrm{~Hz}, \mathrm{C}-1 \beta$ ), 92.7 (dd, $\left.{ }^{2} J_{\mathrm{CF}}=36.6,26.3 \mathrm{~Hz}, \mathrm{C}-1 \alpha\right), 73.6\left(\mathrm{~d},{ }^{3} J_{\mathrm{CF}}=4.4 \mathrm{~Hz}, \mathrm{C}-5 \beta\right), 68.4\left(\mathrm{~d},{ }^{3} J_{\mathrm{CF}}=2.9 \mathrm{~Hz}, \mathrm{C}-5 \alpha\right), 60.5(\mathrm{C}-6 \alpha)$, 60.2 (C-6ß), 59.8 (dd, ${ }^{2} J_{\mathrm{CF}}=30.7,19.0 \mathrm{~Hz}, \mathrm{C}-4 \alpha$ ), 59.5 (dd, ${ }^{2} J_{\mathrm{CF}}=29.3,17.6 \mathrm{~Hz}, \mathrm{C}-4 \beta$ ), 57.4 $\left(\mathrm{C}_{\mathrm{q}, \mathrm{tBu}}, \beta\right), 57.4\left(\mathrm{C}_{\mathrm{q}, \mathrm{tBu}}, \alpha\right), 22.8\left(6 \times \mathrm{CH}_{3, \mathrm{tBu}}, \alpha+\beta\right) \mathrm{ppm}\left(2 \times \mathrm{CF}_{2}, \alpha+\beta\right.$ not visible). ${ }^{19} \mathrm{~F}$ NMR (376 $\mathrm{MHz}, \mathrm{CD}_{3} \mathrm{OD}$ ) $\delta-116.6$ (ddtd, $\left.{ }^{2} J_{\mathrm{FF}}=260.5, J=15.2,9.1,2.2 \mathrm{~Hz}, \mathrm{F \alpha}\right),-118.2\left(\mathrm{~m},{ }^{2} J_{\mathrm{FF}}=261.8 \mathrm{~Hz}\right.$, $\mathrm{F} \beta),-119.1$ (dddd, $\left.{ }^{2} J_{\mathrm{FF}}=269.6, J=19.5,9.5,9.1 \mathrm{~Hz}, \mathrm{F \alpha}\right),-126.3\left(\mathrm{~m},{ }^{2} J_{\mathrm{FF}}=260.5 \mathrm{~Hz}, \mathrm{F \alpha}\right),-128.9$ $\left(\mathrm{m},{ }^{2} J_{\mathrm{FF}}=261.8 \mathrm{~Hz}, \mathrm{~F} \beta\right.$ ), -134.6 (dddd, $\left.{ }^{2} J_{\mathrm{FF}}=269.6, J=16.0,11.3,5.2 \mathrm{~Hz}, \mathrm{F \alpha}\right),-137.4(\mathrm{~m}$, 
$\left.{ }^{2} J_{\mathrm{FF}}=263.1 \mathrm{~Hz}, \mathrm{~F} \beta\right),-138.5\left(\mathrm{dddd},{ }^{2} J_{\mathrm{FF}}=263.1, \mathrm{~J}=17.8,14.3,6.9 \mathrm{~Hz}, \mathrm{~F} \beta\right) \mathrm{ppm} . \mathrm{MS}(\mathrm{ESI}+)(\mathrm{m} / \mathrm{z})$ $387(\mathrm{M}+\mathrm{Na}+\mathrm{MeCN})^{+}$. HRMS $(\mathrm{MS}+)$ for $\mathrm{C}_{10} \mathrm{H}_{17} \mathrm{~F}_{4} \mathrm{NNaO}_{4} \mathrm{~S}(\mathrm{M}+\mathrm{Na})^{+}$calcd 346.0707, found 346.0713.

Analytical sample of the pure diol $\left(2 S, 3 S, R_{S}\right)$-3-(tert-Butylsulfinylamino)-4,4,5,5-tetrafluorohept-

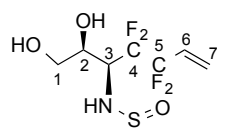

$20 t$ - $t$

6-ene-1,2-diol (20) was obtained by column chromatography (petroleum ether 40-60 ${ }^{\circ} \mathrm{C} /$ acetone 70:30). $\mathrm{R}_{\mathrm{f}} 0.25$ (petroleum ether 40-60 ${ }^{\circ} \mathrm{C} /$ acetone 60:40). $[\alpha]_{D}-44.8\left(\mathrm{c} 0.532, \mathrm{CHCl}_{3}, 21^{\circ} \mathrm{C}\right)$. IR (neat) $3299(\mathrm{~m}), 2963(\mathrm{w}), 1237(\mathrm{~m})$, 1116 (s), 1028 (s) $\mathrm{cm}^{-1} .{ }^{1} \mathrm{H}$ NMR (400 MHz, CD $\left.{ }_{3} \mathrm{OD}\right) \delta \mathrm{ppm} 6.19-6.03(\mathrm{~m}$, $1 \mathrm{H}, \mathrm{H}-6), 5.87$ (dt, $\left.{ }^{3} J_{\mathrm{HH} \text {,trans }}=17.3,{ }^{4} J_{\mathrm{HF}}=2.3 \mathrm{~Hz}, 1 \mathrm{H}, \mathrm{H}-7_{\text {trans }}\right), 5.77$ (d, ${ }^{3} J_{\mathrm{HH}, \text { cis }}=11.1 \mathrm{~Hz}, 1 \mathrm{H}, \mathrm{H}-7_{\text {cis }}$ ), $4.15\left(\mathrm{dd},{ }^{3} J_{\mathrm{HH}}=8.5,5.9 \mathrm{~Hz}, 1 \mathrm{H}, \mathrm{H}-2\right), 4.03\left(\mathrm{t},{ }^{3} J_{\mathrm{HF}}=13.6 \mathrm{~Hz}, 1 \mathrm{H}, \mathrm{H}-3\right), 3.62\left(\mathrm{dd},{ }^{2} J_{\mathrm{HH}}=10.9\right.$, $\left.{ }^{3} J_{\mathrm{HH}}=8.5 \mathrm{~Hz}, 1 \mathrm{H}, \mathrm{H}-1_{\mathrm{a}}\right), 3.54\left(\mathrm{dd},{ }^{2} J_{\mathrm{HH}}=10.9,{ }^{3} J_{\mathrm{HH}}=5.9 \mathrm{~Hz}, 1 \mathrm{H}, \mathrm{H}-1_{\mathrm{b}}\right), 1.26(\mathrm{~s}, 9 \mathrm{H}) .{ }^{13} \mathrm{C} \mathrm{NMR}(101$ $\left.\mathrm{MHz}, \mathrm{CD}_{3} \mathrm{OD}\right) \delta 128.5$ (t, $\left.{ }^{2} J_{\mathrm{CF}}=24.2 \mathrm{~Hz}, \mathrm{C}-6\right), 124.8$ (t, ${ }^{3} J_{\mathrm{CF}}=9.5 \mathrm{~Hz}, \mathrm{C}-7$ ), 118.1 (tt, ${ }^{1} J_{\mathrm{CF}}=255.4$, $\left.{ }^{2} J_{\mathrm{CF}}=33.7 \mathrm{~Hz}, \mathrm{CF}_{2}\right), 117.2\left(\mathrm{tt},{ }^{1} J_{\mathrm{CF}}=248.8,{ }^{2} J_{\mathrm{CF}}=34.4 \mathrm{~Hz}, \mathrm{CF}_{2}\right), 68.4(\mathrm{C}-2), 63.2(\mathrm{C}-1), 58.6(\mathrm{t}$, $\left.{ }^{2} J_{\mathrm{CF}}=22.7 \mathrm{~Hz}, \mathrm{C}-3\right), 58.3\left(\mathrm{C}_{\mathrm{q}, \mathrm{tBu}}\right), 23.1\left(\mathrm{CH}_{3, \mathrm{tBu}}\right) \mathrm{ppm} .{ }^{19} \mathrm{~F} \mathrm{NMR}\left(282 \mathrm{MHz}, \mathrm{CD}_{3} \mathrm{OD}\right) \delta-112.8$ (dd, $\left.{ }^{2} J_{\mathrm{FF}}=265.7,11.8 \mathrm{~Hz}, 1 \mathrm{~F}\right),-113.8\left(\mathrm{dd},{ }^{2} J_{\mathrm{FF}}=265.7,10.7 \mathrm{~Hz}, 1 \mathrm{~F}\right),-117.2\left(\mathrm{dd},{ }^{2} J_{\mathrm{FF}}=274.0,14.0 \mathrm{~Hz}\right.$, $1 \mathrm{~F}),-119.3\left(\mathrm{dd},{ }^{2} J_{\mathrm{FF}}=274.0,12.9 \mathrm{~Hz}, 1 \mathrm{~F}\right) \mathrm{ppm}$. MS (ESI+) $(\mathrm{m} / \mathrm{z}) 385(\mathrm{M}+\mathrm{Na}+\mathrm{MeCN})^{+}$. HRMS $(\mathrm{MS}+)$ for $\mathrm{C}_{11} \mathrm{H}_{19} \mathrm{~F}_{4} \mathrm{NNaO}_{3} \mathrm{~S}(\mathrm{M}+\mathrm{Na})^{+}$calcd 344.0914, found 344.0909.

\subsection{4-Amino-2,3,4-trideoxy-2,2,3,3-tetrafluoro-D-threo-hexopyranose hydrochloride $(\mathbf{8} \cdot \mathrm{HCl})$}

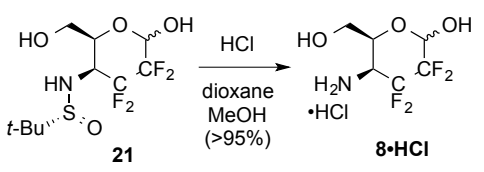

A solution of sulfinamide 21 (190 mg, $0.588 \mathrm{mmol}, 1$ equiv) in $\mathrm{MeOH}(1.2 \mathrm{~mL})$ and $4 \mathrm{M} \mathrm{HCl}$ in dioxane $(0.60 \mathrm{~mL}, 2.35 \mathrm{mmol}, 4$ equiv) was stirred at $\mathrm{rt}$ for $0.5 \mathrm{~h}$ then evaporated in vacuo. The residue was coevaporated with $\mathrm{MeOH}(10 \times 20 \mathrm{~mL})$ then diluted in $\mathrm{H}_{2} \mathrm{O}(15 \mathrm{~mL})$, washed with $\mathrm{Et}_{2} \mathrm{O}(2 \times 5 \mathrm{~mL})$ and concentrated to afford $153 \mathrm{mg}$ of the amine hydrochloride $8 \cdot \mathbf{H C l}$ along with less than $3 \%$ of impurities as a colourless oil. Anomeric ratio at equilibrium in $\mathrm{CD}_{3} \mathrm{OD}$ : $54: 46$ $\alpha / \beta$. Approximated yield >95\%. IR (neat) $3210(\mathrm{~m}, \mathrm{br}), 2886(\mathrm{~m}), 1526(\mathrm{~m}), 1109(\mathrm{~s}), 1029(\mathrm{~s})$ $\mathrm{cm}^{-1}$. ${ }^{1} \mathrm{H}$ NMR $\left(400 \mathrm{MHz}, \mathrm{CD}_{3} \mathrm{OD}\right) \delta 5.39(\mathrm{dd}, \mathrm{J}=8.5,7.1 \mathrm{~Hz}, 1 \mathrm{H}, \mathrm{H}-1 \alpha), 5.12(\mathrm{dd}, J=15.0,3.1 \mathrm{~Hz}$,

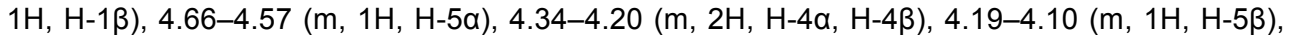
3.96-3.71 ppm (m, 4H, $2 \times \mathrm{H}-6 \alpha, 2 \times \mathrm{H}-6 \beta)$ ppm. ${ }^{13} \mathrm{C}$ NMR (101 MHz, CD $\left.{ }_{3} \mathrm{OD}\right) \delta 117.5-108.7$ $\left(2 \times \mathrm{CF}_{2}, \alpha+\beta\right), 93.5\left(\mathrm{ddd},{ }^{2} J_{\mathrm{CF}}=26.4,19.0,{ }^{3} J_{\mathrm{CF}}=3.4 \mathrm{~Hz}, \mathrm{C}-1 \beta\right), 92.9$ (dd, ${ }^{2} J_{\mathrm{CF}}=36.7,24.9 \mathrm{~Hz}, \mathrm{C}-$ 


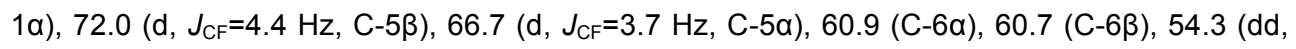
$\left.{ }^{2} J_{\mathrm{CF}}=33.4,19.4 \mathrm{~Hz}, \mathrm{C}-4 \alpha\right), 53.9$ (dd, $\left.{ }^{2} J_{\mathrm{CF}}=32.6,19.4 \mathrm{~Hz}, \mathrm{C}-4 \beta\right) \mathrm{ppm} .{ }^{19} \mathrm{~F}$ NMR (376 MHz, $\mathrm{CD}_{3} \mathrm{OD}$ ) $\delta-116.9$ (app. ddt, $\left.{ }^{2} J_{\mathrm{FF}}=274.0, \mathrm{~J}=15.9,9.2, \mathrm{~Hz}, \mathrm{F \alpha}\right),-118.1--118.9$ (m, F $),-119.5$ $\left(\mathrm{ddt},{ }^{2} J_{\mathrm{FF}}=273.8,{ }^{3} J_{\mathrm{FF}}=17.5,{ }^{3} J_{\mathrm{FF}}=8.7, J_{\mathrm{HF}}=8.7 \mathrm{~Hz}, \mathrm{F \alpha}\right),-125.3--126.3(\mathrm{~m}, \mathrm{F \alpha}),-127.8--128.7$ $(\mathrm{m}, \mathrm{F} \beta),-136.0$ (dddd, ${ }^{2} J_{\mathrm{FF}}=273.8,{ }^{3} J_{\mathrm{FF}}=16.2,{ }^{3} J_{\mathrm{FF}}=10.3, J_{\mathrm{HF}}=4.2 \mathrm{~Hz}, \mathrm{F \alpha}$ ), -137.9 (app. dtd, $\left.{ }^{2} J_{\mathrm{FF}}=267.5, J=15.4,6.6 \mathrm{~Hz}, \mathrm{~F} \beta\right),-139.2\left(\mathrm{~m},{ }^{2} J_{\mathrm{FF}}=267.5 \mathrm{~Hz}, \mathrm{~F} \beta\right) \mathrm{ppm} .\left\{{ }^{1} \mathrm{H}\right\}{ }^{19} \mathrm{~F} \mathrm{NMR}(376 \mathrm{MHz}$, $\left.\mathrm{CD}_{3} \mathrm{OD}\right) \delta-116.8$ (ddd, $\left.{ }^{2} J_{\mathrm{FF}}=274.0,{ }^{3} J_{\mathrm{FF}}=16.1,{ }^{3} J_{\mathrm{FF}}=8.6 \mathrm{~Hz}, \mathrm{F \alpha}\right),-118.5$ (ddd, ${ }^{2} J_{\mathrm{FF}}=275.0$, ${ }^{3} J_{\mathrm{FF}}=13.4,{ }^{3} J_{\mathrm{FF}}=6.4 \mathrm{~Hz}, \mathrm{~F} \beta$ ), -119.4 (ddd, ${ }^{2} J_{\mathrm{FF}}=273.9,{ }^{3} J_{\mathrm{FF}}=17.3,{ }^{3} J_{\mathrm{FF}}=8.5 \mathrm{~Hz}, \mathrm{F \alpha}$ ), -125.8 (ddd,

$\left.{ }^{2} J_{\mathrm{FF}}=274.0,{ }^{3} J_{\mathrm{FF}}=17.3,{ }^{3} J_{\mathrm{FF}}=10.3 \mathrm{~Hz}, \mathrm{F \alpha}\right),-128.3\left(\mathrm{ddd},{ }^{2} J_{\mathrm{FF}}=275.2,{ }^{3} J_{\mathrm{FF}}=15.6,{ }^{3} J_{\mathrm{FF}}=10.5 \mathrm{~Hz}, \mathrm{~F} \beta\right.$ ), $-136.0\left(\mathrm{ddd},{ }^{2} J_{\mathrm{FF}}=273.8,{ }^{3} J_{\mathrm{FF}}=16.1,{ }^{3} J_{\mathrm{FF}}=10.3 \mathrm{~Hz}, \mathrm{F \alpha}\right),-138.3--137.5(\mathrm{~m}, \mathrm{~F} \beta),-139.2$ (ddd, $\left.{ }^{2} J_{\mathrm{FF}}=267.5,{ }^{3} J_{\mathrm{FF}}=13.4,{ }^{3} J_{\mathrm{FF}}=10.5 \mathrm{~Hz}, \mathrm{~F} \beta\right) \mathrm{ppm}$. MS (ESI+) $(\mathrm{m} / \mathrm{z}) 220(\mathrm{M}+\mathrm{H})^{+}$. HRMS (MS+) for $\mathrm{C}_{6} \mathrm{H}_{10} \mathrm{~F}_{4} \mathrm{NO}_{3}(\mathrm{M}+\mathrm{H})^{+}$calcd 220.0591, found 220.0596.

Supporting information General information, copies of ${ }^{1} \mathrm{H},{ }^{13} \mathrm{C}$, and ${ }^{19} \mathrm{~F}$ NMR spectra of all compounds, and HMBC spectra of 7 and 8.

Acknowledgements The European Community (INTERREG IVa Channel Programme, IS:CEChem, project 4061) is thanked for financial support.

[1] (a) Glaudemans, C. P. J. Chem. Rev. 1991, 91, 25-33. (b) Lemieux, R. U. Chem. Soc. Rev. 1989, 18, 347-374.

| [2] (a) Kim, J.-H.; Resende, R.; Wennekes, T.; Chen, H.-M.; Bance, N.; Buchini, S.; Watts, A. G.; Pilling, P.; Streltsov, V. A.; Petric, M.; Liggins, R.; Barrett, S.; McKimm-Breschkin, J. L.; Niikura, M.; Withers, S. G. Science, 2013, 340, 71-75; (b) Vocadlo, D.; Davies, G. J.; Laine, R.; Withers, S. G. Nature, 2001, 421, 835-838. Reviews: (c) Zechel, D. L.; Withers, S. G. Acc. Chem. Res. 2000, 33, 11-18. (d) Pongdee, R.; Liu, H.-W. Bioorg. Chem. 2004, 32, 393-437.

[3] Wagner, S.; Mersch, C.; Hoffmann-Röder, A. Chem. Eur. J. 2010, 16, 7319-7330.

[4] Reviews: (a) Cheng, Y.; Guo, A.-L.; Guo, D.-S. Curr. Org. Chem. 2010, 14, 977-999. (b) Hein, M.; Miethchen, R. Adv. Org. Synth. 2006, 2, 381-429. (c) Miethchen, R. J. Fluorine Chem. 2004, 125, 895-901. (d) Dax, K.; Albert, M.; Ortner, J.; Paul, B. J. Carbohydr. Res. 2000, 327, 47-86. (e) Dax, K.; Albert, M.; Ortner, J.; Paul, B. J. Curr. Org. Chem. 1999, 3, 287-307. (f) Tsuchiya, T. Adv. Carbohydr. Chem. Biochem. 1990, 48, 91-277. (g) Card, P. J. J. Carbohydr. Chem. 1985, 4, 451-487. (h) Penglis, A. A. E. Adv. Carbohydr. Chem. Biochem. 1981, 38, 195285.

| [5] Graton, J.; Wang, Z.; Brossard, A.-M.; Goncalves Monteiro, D.; Le Questel, J.-Y.; Linclau, B. Angew. Chem. Int. Ed. 2012, 51, 6176-6180.

| [6] Laurence, C.; Brameld, K. A.; Graton, J.; Le Questel, J.-Y.; Renault, E. J. Med. Chem. 2009, 52, 4073-4086.

| [7] (a) Bernardi A.; Jiménez-Barbero, J.; Casnati, A.; De Castro, C.; Darbre, T.; Fieschi, F.; Finne, J.; Funken, H.; Jaeger, K.-E.; Lahmann, M.; Lindhorst, T. K.; Marradi, M.; Messner, P.; 
Molinaro, A.; Murphy, P. V.; Nativi, C.; Oscarson, S.; Penadés, S.; Peri, F.; Pieters, R. J.; Renaudet, O.; Reymond, J.-L.; Richichi, B.; Rojo, J.; Sansone, F.; Schäffer, C.; Turnbull, W. B.; Velasco-Torrijos, T.; Vidal, S.; Vincent, S.; Wennekes, T.; Zuilhof, H.; Imberty, A. Chem. Soc. Rev. 2013, 42, 4709-4727; (b) Ernst, B.; Magnani, J. L. Nat. Rev. Drug Discov. 2009, 8, 661677. (c) Koester, D. C.; Holkenbrink A.; Werz, D. B. Synthesis 2010, 3217-3242; (d) Sears, P.; Wong, C.-H. Angew. Chem. Int. Ed. 1999, 38, 2300-2324.

| 8] (a) Dalvi, V. H.; Rossky, P. J. Proc. Nat. Acad. USA. 2010, 107, 13603-13607; (b) Mecinovic, J.; Snyder, P. W.; Mirica, K. A.; Bai, S.; Mack, E. T.; Kwant, R. L.; Moustakas, D. T.; Heroux A.; Whitesides, G. M. J. Am. Chem. Soc. 2011, 133, 14017-14026.

| 9] Müller, K.; Faeh, C. ; Diederich, F. Science 2007, 317, 1881-1886; (b) Zürcher, M. ; Diederich, F. J. Org. Chem. 2008, 73, 4345-4361.

| [10] (a) Kim, H. W.; Rossi, P.; Schoemaker, R. K.; DiMagno, S. G. J. Am. Chem. Soc. 1998, 120, 9082-9083; (b) Biffinger, J. C.; Kim, H. W.; DiMagno, S. G. ChemBioChem. 2004, 5, 622627.

11] Timofte, R. S Linclau, B. Org. Lett. 2008, 10, 3673-3676

[12] (a) Linclau, B.; Golten, S.; Light, M.; Sebban, M.; Oulyadi, H. Carbohydr. Res. 2011, 346, 1129-1139; (b) Linclau, B.; Golten, S.; Light, M. J. Carbohydrate Chem. 2011, 60, 618-625.

| [13] loannou, A.; Cini, E.; Timofte, R. S.; Flitsch, S. L.; Turner, N. J.; Linclau, B. Chem. Commun. 2011, 47, 11228-11230.

| [14] N'Go, I.; Golten, S.; Arda, A.; Canada, J.; Jimenez-Barbero, J.; Linclau, B.; Vincent, S. Chem. Eur. J. 2014, 20, 106-112.

| [15] Reviews: (a) Pfrengle, F.; Reissig, H.-U. Chem. Soc. Rev. 2010, 39, 549-557. (b) Rai, R.; McAlexander, I.; Chang, C.-W. T. Org. Prep. Proc. Int. 2005, 37, 337-375.

| [16] Examples: (a) Albler, C.; Schmid, W. Eur. J. Org. Chem. 2014, 2451-2459; (b) Poirot, E.; Chang, A. H. C.; Horton, D.; Kovac, P. Carbohydrate Res. 2001, 334, 195-205.

[17] Review: Robak, M. T.; Herbage, M. A.; Ellman, J. A. Chem. Rev. 2010, 110, 3600-3740.

[18] Examples; (a) Buesking, A. W.; Baguley, T. D.; Ellman, J. A. Org. Lett. 2011, 13, 964-967. (b) Fontenelle, C. Q.; Conroy, M.; Light, M.; Poisson, T.; Pannecoucke, X.; Linclau, B. J. Org. Chem. 2014, 79, 4186-4195.

| [19] Konno, T.; Takano, S.; Takahashi Y.; Konishi, H.; Tanaka, Y.; Ishihara T. Synthesis 2011, 33-44.

| [20] Konno, T.; Hoshino, T.; Kida, T.; Takano, S.; Ishihara T. J. Fluorine Chem. 2013, 152, 106113.

[21] Bergeron, M.; Johnson, T.; Paquin, J.-F. Angew. Chem. Int. Ed. 2011, 50, 11112-11116.

[22] Wray, V. J. Chem. Soc., Perkin Trans. II 1976, 1598-1605.

[23] This is in contrast with the stereoselection as assumed in ref 19

[24] (a) Evans, D. A.; Siska, S. J.; Cee, V. J. Angew. Chem. 2003, 115, 1803-1807; Angew. Chem. Int. Ed. 2003, 42, 1761-1765; (b) Cee, V. J.; Cramer, C. J.; Evans, D. A. J. Am. Chem. Soc. 2006, 128, 2920-2930.

| [25] (a) Cherest, M.; Felkin, H.; Prudent, N. Tetrahedron Lett. 1968, 9, 2199-2204; (b) Anh, N. T. Eisenstein, O. Nouv. J. Chim. 1977, 1, 61-70.

[26] Other recent examples of double diastereodifferentiation, see refs 17 and 18. 